\title{
Renal cell-expressed TNF receptor 2, not receptor 1 , is essential for the development of glomerulonephritis
}

\author{
Volker Vielhauer, George Stavrakis, and Tanya N. Mayadas
}

Center of Excellence in Vascular Biology, Department of Pathology, Brigham and Women's Hospital and Harvard Medical School, Boston, Massachusetts, USA.

\begin{abstract}
TNF is essential for the development of glomerulonephritis, an immune-mediated disorder that is a major cause of renal failure worldwide. However, TNF has proinflammatory and immunosuppressive properties that may segregate at the level of the 2 TNF receptors (TNFRs), TNFR1 and TNFR2. TNFR1-deficient mice subjected to immune complex-mediated glomerulonephritis developed less proteinuria and glomerular injury, and fewer renal leukocyte infiltrates at early time points after disease induction, and this was associated with a reduced systemic immune response to nephrotoxic rabbit IgG. However, proteinuria and renal pathology were similar to those in wild-type controls at later time points, when lack of TNFR1 resulted in excessive renal $T$ cell accumulation and an associated reduction in apoptosis of these cells. In sharp contrast, TNFR2-deficient mice were completely protected from glomerulonephritis at all time points, despite an intact systemic immune response. TNFR2 was induced on glomerular endothelial cells of nephritic kidneys, and TNFR2 expression on intrinsic cells, but not leukocytes, was essential for glomerulonephritis and glomerular complement deposition. Thus, TNFR1 promotes systemic immune responses and renal $T$ cell death, while intrinsic cell TNFR2 plays a critical role in complement-dependent tissue injury. Therefore, therapeutic blockade specifically of TNFR2 may be a promising strategy in the treatment of immune-mediated glomerulonephritis.
\end{abstract}

\section{Introduction}

Progressive glomerulonephritis (GN) occurs in a variety of human immune-mediated disorders, including anti-glomerular basement membrane (anti-GBM) disease, systemic vasculitis, and systemic lupus erythematosus $(1,2)$. Most forms of GN are characterized by glomerular deposition or in situ formation of immune complexes, which initiate a humoral and cellular immune response. The resulting glomerular injury leads to proteinuria and deterioration of renal function. Progressive forms of GN are histologically characterized by mononuclear leukocyte infiltration in the glomeruli, glomerular cell proliferation, matrix deposition, crescent formation, and eventually glomerulosclerosis. The mechanisms controlling the initiation and progression of GN are not well understood. There is increasing evidence that chemokines and cytokines play a central role in the pathogenesis of GN (3).

Levels of TNF, a potent proinflammatory cytokine, are significantly increased in the kidneys of patients with inflammatory renal diseases such as GN. Furthermore, the functional role of TNF in GN has been demonstrated in animal models. Systemic administration of TNF induces glomerular damage in rabbits (4) and exacerbates glomerular injury in nephrotoxic nephritis in rats (5). TNF-deficient mice subjected to nephrotoxic nephritis demonstrate a partial reduction in proteinuria, glomerular crescent formation, infiltration of leukocytes, and expression of vascular adhesion molecules $(6,7)$. Anti-TNF antibodies attenuate acute glomerular injury in anti-GBM antibody-induced GN in rats (8),

Nonstandard abbreviations used: GBM, glomerular basement membrane; GN, glomerulonephritis; PECAM-1, platelet-endothelial cell adhesion molecule-1; TNFR, TNF receptor

Conflict of interest: The authors have declared that no conflict of interest exists.

Citation for this article: J. Clin. Invest. 115:1199-1209 (2005).

doi:10.1172/JCI200523348 and administration of soluble TNF receptors (TNFRs), which neutralize biological TNF activity, reduces glomerular lesions and prevents crescent formation in rats developing crescentic GN $(9,10)$. TNF is produced predominantly by macrophages and $\mathrm{T}$ cells, but neutrophils, mast cells, and endothelium also express it (11). Furthermore, intrinsic renal cells, including mesangial, glomerular, and tubular epithelial cells produce TNF upon injury or stimulation (12-16). Indeed, TNF produced by intrinsic renal cells but not infiltrating leukocytes contributes to the development of GN (17). TNF induces the expression of major histocompatibility complex class I and II molecules, upregulates endothelial adhesion molecules, and increases the procoagulant activity of endothelium. Moreover, it stimulates the release of other proinflammatory chemokines and cytokines $(18,19)$. However, in addition to its proinflammatory responses, TNF also has immunosuppressive effects. For example, TNF induces apoptosis of activated $\mathrm{T}$ cells and promotes regression of autoimmune reactivity (20-22). Indeed, the importance of understanding the molecular basis of the contrasting effects of TNF is underscored by reports that anti-TNF therapies in patients with rheumatoid arthritis and inflammatory bowel disease induce autoimmune symptoms in some patients, including the development of autoantibodies and lupus-like syndromes (21).

The biological activities of TNF are mediated by 2 functionally distinct TNFRs, the 55-kDa TNFR1 (p55, CD120a) and the 75-kDa TNFR2 (p75, CD120b), which are widely expressed on various cell types in vitro, including leukocytes and endothelial cells. In human kidney, TNFR1 is present in normal glomerular endothelium, where it is primarily localized within the Golgi apparatus (23), and it is strongly expressed in glomeruli of patients with proliferative lupus nephritis (24). Glomerular TNFR2 expression was found in various glomerular diseases and in tubular epithelial cells during acute transplant rejection $(23,24)$. Many of the proinflammatory 


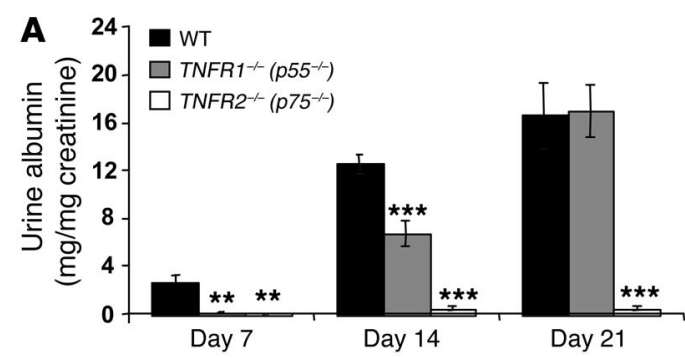

B

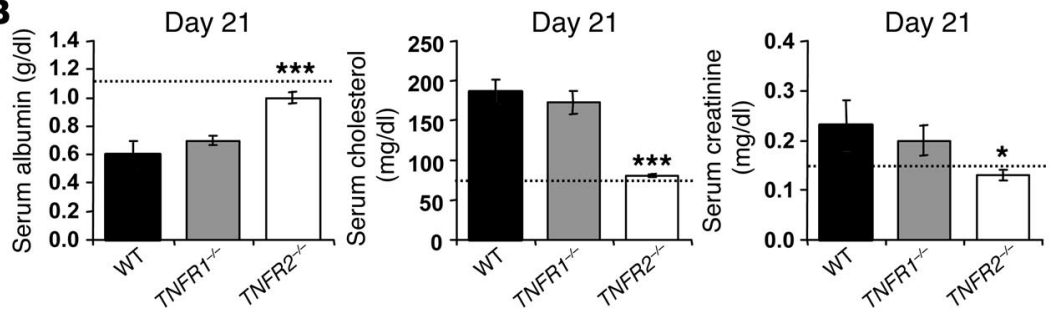

\section{Figure 1}

Wild-type and TNFR1-deficient, but not TNFR2-deficient, mice develop albuminuria with hypoalbuminemia, hypercholesterolemia, and elevated serum creatinine levels at day 21. (A) Albuminuria was evaluated at indicated days after injection of nephrotoxic serum ( $n=9$ per group). (B) Serum levels for albumin, cholesterol, and creatinine were determined at day 21 ( $n=9$ per group). The dotted lines indicate baseline values observed in normal wild-type mice. Data represent the mean \pm SEM. ${ }^{*} P<0.05$, ${ }^{* *} P<0.01$, and ${ }^{* *} P<0.001$ compared with wild-type. actions of TNF are mediated by TNFR1 $(25,26)$, but in several cases TNFR1 activation requires the coordinate expression of TNFR2, which can form heterocomplexes with TNFR1 (27-29). TNFR2 may also potentiate responses mediated by TNFR1 by increasing the local concentration of surface-associated TNF available to TNFR1 (30). Ligation of TNFR2 alone mediates cell proliferation and upregulation of vascular adhesion molecules $(31,32)$.

We investigated the specific role of TNFR 1 and TNFR 2 in mediating renal injury during accelerated nephrotoxic nephritis, a model of immune complex GN induced by anti-GBM antibody and characterized by prolonged proteinuria, glomerular damage, leukocyte infiltration, and renal functional impairment $(33,34)$. Our results demonstrate that TNFR1 accelerates the systemic immune response toward the glomerular immune complexes and that its absence results in increased renal $\mathrm{T}$ cell accumulation, which is associated with a reduction in $\mathrm{T}$ cell apoptosis. However, TNFR1 expression on cells other than T cells is responsible for this phenotype, and overall, TNFR1 function is not essential for the development of GN. In contrast, TNFR2 expression by intrinsic cells is essential for the development of GN, possibly through the modulation of glomerular complement deposition. Furthermore, TNFR2 expression is induced on glomerular endothelial cells of nephritic kidneys, which suggests a role for TNFR2 expression on this cell type in disease progression. These data indicate that TNFR1 and TNFR2 have contrasting roles in GN and suggest that specific blockade of TNFR2, rather than $\mathrm{TNF}$, may represent a promising therapeutic target in the treatment of GN.

\section{Results}

TNFR2, but not TNFR1, is required for the development of proteinuria and glomerular injury. To understand the functional role of the 2 TNFRs in GN, we analyzed a T cell-dependent model of GN $(33,34)$ in TNFR1- and TNFR2-deficient mice. Albuminuria and serum parameters were determined from urine and blood samples taken at periodic time points, and a histologic evaluation of

\section{Table 1} wild-type mice.
Glomerular injury, glomerular deposition of autologous IgG and complement, and renal leukocyte infiltrates at day 21 after induction of nephrotoxic serum nephritis ${ }^{A}$

\section{Day 21}

Glomerular PAS deposition (score)

Glomeruli with crescents (\%)

Autologous IgG end point titer (glomerulus)

Complement C3 deposition (glomerulus)

CD3 $+T$ cells (glomerulus)

$\mathrm{CD}^{+} \mathrm{T}$ cells/HPF (interstitial)

ER-HR3+ macrophages (glomerulus)

ER-HR3 ${ }^{+}$macrophages/HPF (interstitial) glomeruli was performed 21 days after induction of nephritis. Wild-type mice developed progressive albuminuria from day 7 to 21 (Figure 1A). TNFR1deficient mice had minimal albuminuria at day 7 , but by day 21 albuminuria in TNFR1-deficient mice was similar to that in wild-type animals (Figure 1A). In contrast, TNFR2-deficient mice exhibited minimal albuminuria throughout the study period. Serum ypoalbuminemia and hypercholesterolemia were observed in wildand TNFR1-deficient mice, which is consistent with the develbserved in unteated wild-type mice (Figure 1B). in levels were elevated in wild-type and TNFR1-deficient in TNFR2-deficient mice (Figure 1B). Histologica TNFR1-deficient mice (Figure 2, A and B and Table 1), although lesions were less severe in TNFR1-deficient mice than in wild-type TNFR2-deficient mice (Figure 2C and Table 1).

The immune response to deposited immune complexes was evaluated. Equivalent deposition of autologous mouse antibody in glomeruli of wild-type, TNFR1-, and TNFR2-deficient mice was essed by the mean immunofluorescence end point titer required ing mouse anti-tis mice by $38 \%$ (Figure 3), while serum IgG of TNFR2-deficient mice showed a consistent trend toward higher titers compared with wild-type counterparts (Figure 3 ). These findings demonstrate a
AValues are mean $\pm \mathrm{SD} ; n=9$ per group. ${ }^{\mathrm{B}} P<0.05$, $\mathrm{C} P<0.001$, and $\mathrm{D} P<0.01$ compared with 

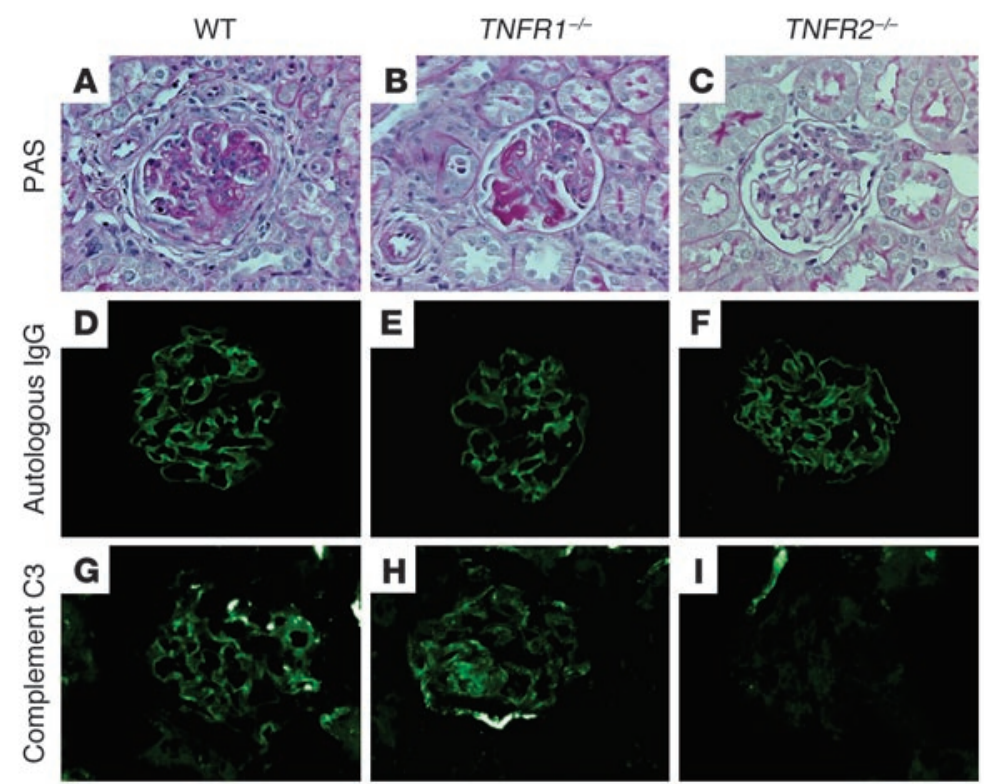

\section{Figure 2}

Glomerular injury and glomerular deposition of autologous mouse anti-rabbit antibody and complement in nephritic wildtype, TNFR1-, and TNFR2-deficient mice at day 21 of nephrotoxic nephritis. (A-C) Representative PAS-stained paraffin sections of wild-type, TNFR1-, and TNFR2-deficient mice. (D-F) Demonstration of glomerular mouse anti-rabbit antibody deposition in each genotype after staining of sections with FITC-labeled anti-mouse antibody. (G-I) Glomerular deposition of complement as revealed by staining with a FITC-labeled anti-mouse C3 antibody. Original magnification $(A-1), \times 630$. The results of the corresponding morphometric analysis are shown in Table 1.

neys of TNFR1-deficient mice at days 21 and 42 (Figure 6 ), in both the glomeruli and interstitium, as assessed by immunohistochemistry (Table 2). Thus TNFR1 deficiency delays the onset of disease, but this protective effect is lost at later time points of disease and is associated with excessive renal accumulation of $\mathrm{T}$ cells.

To gain insights into the cell types contributing to the phenotype in TNFR1-deficient mice, we evaluated the

deficiency in the humoral immune response toward the administered rabbit IgG in TNFR1- but not TNFR2-deficient mice. Complement activation is associated with glomerular damage in a number of disease models $(35,36)$. Complement $\mathrm{C} 3$ deposition, observed in glomerular capillary loops of wild-type and TNFR1deficient mice, was significantly reduced in TNFR2-deficient mice (Figure 2, G-I and Table 1).

Renal leukocyte infiltrates are prominent in TNFR1-deficient mice but minimal in TNFR2-deficient mice. Infiltrating renal leukocytes were quantitated by flow cytometric analysis of renal single-cell suspensions and immunohistochemical analysis of tissue sections stained with relevant antibodies. Increased accumulation of $\alpha \beta \mathrm{T}$ cells, $\gamma \delta$ T cells, and macrophages was noted in nephritic wild-type kidneys. In TNFR1-deficient mice, renal macrophage infiltration was similar to that in wild-type mice, but the number of renal $\alpha \beta$ and $\gamma \delta$ T cells was significantly increased, by 2.5 -fold and 3.1-fold, respectively (Figure $4 \mathrm{~A})$. In contrast, renal $\mathrm{T}$ cell and macrophage infiltration was minimal in TNFR2-deficient mice (Figure 4A), which is consistent with the absence of significant proteinuria and glomerular injury in these animals. Immunohistochemical analysis of $\mathrm{CD}^{+} \mathrm{T}$ cells and macrophages in the different sets of animals corroborated these results (Figure 4, B-G and Table 1).

TNFR1 deficiency delays the development of proteinuria and glomerular injury but also results in excessive renal $\mathrm{T}$ cell accumulation. The opposing effects of TNFR1 at early and later stages of GN (Figures 1 and 2) prompted us to extend these data with a more detailed analysis of renal histology and function in TNFR1deficient mice at day 7, 21 (repeat of Figures 1 and 2), and 42 after induction of GN. At day 7, TNFR1-deficient mice developed minimal albuminuria and hypoalbuminemia (Figure 5), no significant elevations in serum cholesterol and creatinine values (Figure 5B), and no glomerular PAS deposition (Table 2). Results at day 21 are those reported in Table 1. At day 42, histological and functional parameters were similar in wild-type and TNFR1-deficient mice (Figure 5 and Table 2). Glomerular deposition of autologous mouse antibody and complement C3 (Table 2) and renal macrophages (Figure 6) was reduced in TNFR1-deficient mice only at day 7. Interestingly, more $\alpha \beta$ and $\gamma \delta$ T cells accumulated in nephritic kidexpression pattern of TNFR1 on circulating, splenic, and renal accumulated leukocytes in nephritic wild-type mice by flow cytometry. TNFR1 was expressed on T cells and macrophages at comparable levels in the peripheral blood and spleen (Figure 7A and Table 3). Renal $\alpha \beta$ T cells and $\gamma \delta$ T cells also expressed TNFR1, although expression levels were significantly lower than in peripheral $\mathrm{T}$ cells, and renal macrophages had little TNFR1 (Figure 7A and Table 3). This could reflect the internalization or shedding of the receptor upon cell activation (37). Given the described role of TNFR1 in T cell apoptosis (22), we investigated whether the enhanced renal $\mathrm{T}$ cell accumulation in TNFR1-deficient mice was associated with a reduction in their apoptosis. Immunostaining for cleaved caspase 3 revealed significantly fewer apoptotic cells in nephritic kidneys of TNFR1-deficient mice than in those of wild-type mice at day 42 after induction of GN $(5.9 \pm 1.3$ vs. $8.4 \pm 2.5$ cells/high-power field [HPF], respectively; $n=7$ per group; $P<0.05$ ). Additionally, flow cytometry performed by staining CD3-, $\alpha \beta$ TCR-positive T cells of selected mice with annexin $\mathrm{V}$ demonstrated a reduction in the number of apoptotic $\alpha \beta$ T cells in spleen, lymph nodes, and kidneys of TNFR1deficient mice compared with wild-type mice (Figure 7B). Thus, the

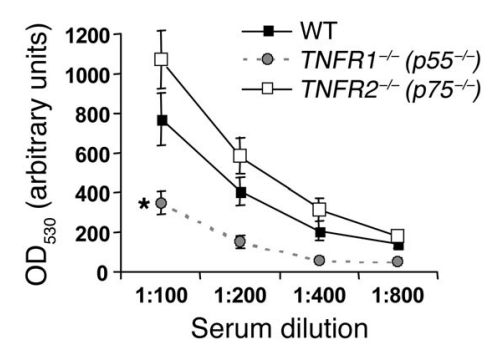

\section{Figure 3}

Circulating autologous mouse anti-rabbit IgG levels in wild-type, TNFR1-, and TNFR2-deficient mice. Antibody levels were determined by ELISA from serum samples of animals at day 21 . Titers in serial dilutions of serum are expressed in arbitrary units ( $n=9$ per group). Data represent the mean \pm SEM. ${ }^{\star} P<0.05$ compared with wild-type. $\mathrm{OD}_{530}$, optical density at $530 \mathrm{~nm}$. 


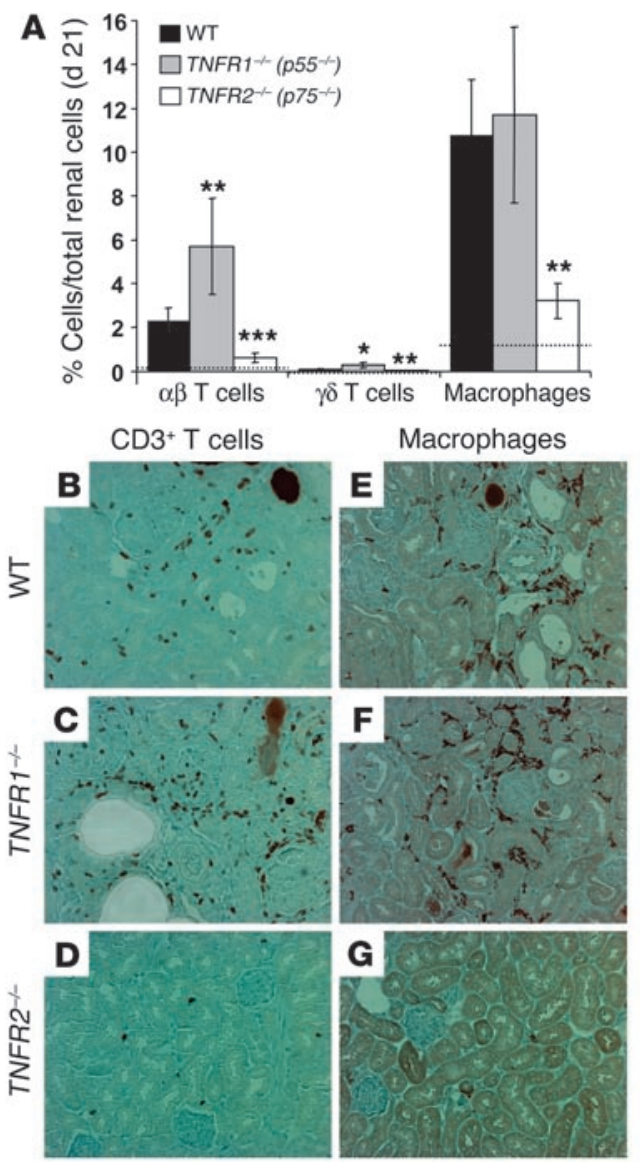

excessive accumulation of renal $\mathrm{T}$ cells may result, at least in part, from a reduction in $\mathrm{T}$ cell apoptosis. To investigate whether TNFR1 on $\mathrm{T}$ cells is responsible for this effect, we reconstituted mice deficient in $\alpha \beta$ T cells with either wild-type or TNFR1-deficient splenic $\alpha \beta$ T cells and subjected them to GN. All disease parameters including renal $\mathrm{T}$ cell accumulation were comparable in the 2 groups of mice at day 42, a time point at which significant differences in $\mathrm{T}$ cell accumulation were observed in WT and TNFR1-deficient mice. Moreover, the number of apoptotic $\alpha \beta \mathrm{T}$ cells was similar in mice with wild-type and TNFR1-deficient T cells (data not shown). Thus, engagement of TNFR 1 on cells other than $\alpha \beta$ T cells is required for increased accumulation and reduced apoptosis of renal $\alpha \beta$ T cell accumulation during GN.

Analysis of TNFR2 expression in nephritic mice. TNFR2 expression in nephritic wild-type mice was examined by flow cytometry. A simi-

\section{Figure 5}

TNFR1-deficient mice are protected from nephritis at day 7 but not at days 21 and 42 after injection of nephrotoxic serum. (A) Albuminuria in TNFR1-deficient mice was absent at day 7 but comparable to that of wild-type at days 14 and 21 ( $n=6-8$ per group). (B) Hypoalbuminemia was absent in TNFR1-deficient mice at day 7 but was comparable to that of wild-type at later time points. Serum levels for cholesterol and creatinine were normal in wild-type and TNFR1-deficient mice at day 7 and were similarly elevated at day 42 ( $n=6-8$ per group). The dotted lines indicate baseline values observed in normal wild-type mice. Data represent the mean \pm SEM. ${ }^{* \star} P<0.01,{ }^{* *} P<0.001$ compared with wild-type.

\section{Figure 4}

Renal leukocyte infiltrates in nephritic wild-type, TNFR1-, and TNFR2deficient mice at day 21 of nephrotoxic nephritis. (A) Single-cell suspensions were prepared from nephritic kidneys. These cells were then stained for CD3 $\varepsilon, \alpha \beta$ TCR, $\gamma \delta$ TCR, and the macrophage marker F4/80 and analyzed by flow cytometry ( $n=8-10$ per group). The dotted lines indicate baseline values observed in normal wild-type mice. Data represent the mean \pm SD. ${ }^{\star} P<0.05,{ }^{* \star} P<0.01,{ }^{* \star \star} P<0.001$ compared with wild-type. (B-D) Representative renal sections of wild-type, TNFR1-, and TNFR2-deficient mice stained for $\mathrm{CD}^{+} \mathrm{T}$ cells and $(\mathrm{E}-\mathrm{G})$ the macrophage marker ER-HR3. Original magnification, $\times 400$. The results of the corresponding morphometric analysis are shown in Table 1.

lar percentage of $\mathrm{T}$ cells and macrophages in the peripheral blood and spleen expressed TNFR2 (Figure 7C and Table 3). In the kidney, a high proportion of renal $\alpha \beta$ T cells were positive for TNFR2, whereas renal $\gamma \delta$ T cells expressed lower levels of TNFR 2 than did $\gamma \delta$ $\mathrm{T}$ cells in peripheral organs. Interestingly, TNFR2 expression was nearly undetectable on renal macrophages (Figure 7C and Table 3).

Immunohistochemical analysis of kidney sections revealed positive staining for TNFR2 in glomeruli and in postcapillary venules of the cortex (Figure $8 \mathrm{~B}$ ). Importantly, kidneys of control mice without $\mathrm{GN}$ exhibited no specific staining for the receptor (Figure 8A). Furthermore, the cellular pattern of TNFR2 expression in the kidney was highly disease specific. That is, while a glomerular/venular pattern of staining was observed in nephritic kidneys, TNFR2 staining was restricted to tubular epithelial cells following obstructive nephropathy induced by unilateral ureteral ligation, which results primarily in tubular epithelial cell damage (Figure 8C). Confocal imaging was undertaken to identify the resident glomerular cell type expressing TNFR2 following GN induction. TNFR2 staining was observed in glomerular capillaries and colocalized with positive signals for CD31/platelet-endothelial cell adhesion molecule-1 (PECAM-1), an endothelial cell marker (Figure 8, D-F). Thus, nephritis induces glomerular endothelial cell expression of TNFR2.
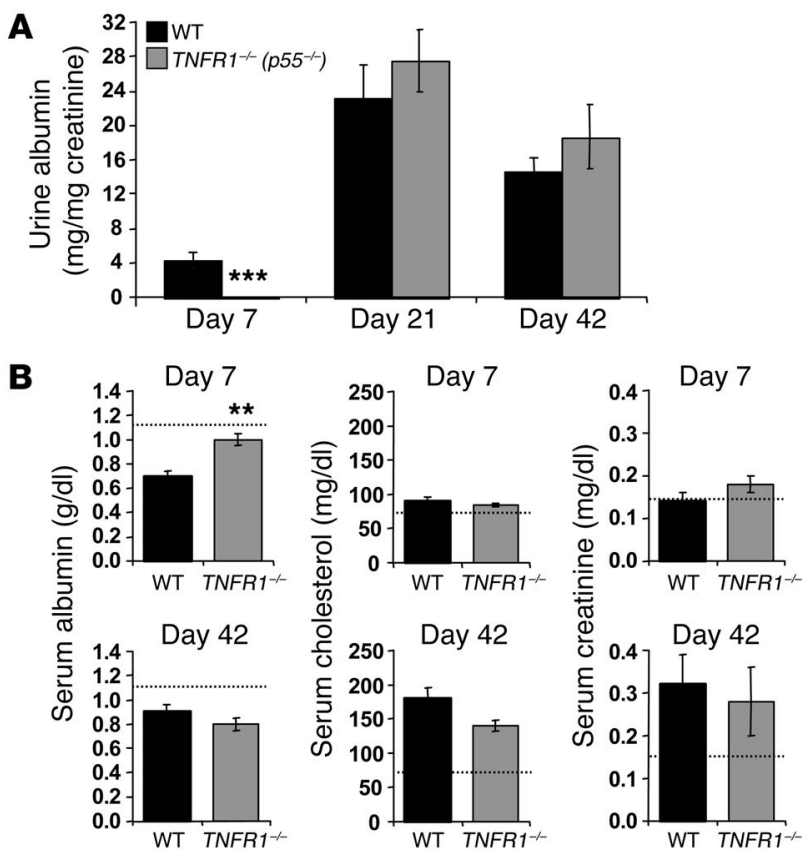


\section{Table 2}

Evaluation of glomerular injury, glomerular deposition of autologous $\lg G$ and complement, and renal leukocyte infiltrates in wild-type and TNFR1-deficient mice at days 7, 21, and 42 after induction of nephrotoxic serum nephritis ${ }^{A}$

\begin{tabular}{|c|c|c|c|c|c|c|}
\hline & \multicolumn{2}{|c|}{ Day 7} & \multicolumn{2}{|c|}{ Day 21} & \multicolumn{2}{|c|}{ Day 42} \\
\hline & Wild-type & TNFR1-/- & Wild-type & TNFR1-/- & Wild-type & TNFR1 $1^{--}$ \\
\hline Glomerular PAS deposition (score) & $0.26 \pm 0.18$ & $0.07 \pm 0.04^{B}$ & $1.10 \pm 0.16$ & $0.75 \pm 0.27^{\mathrm{B}}$ & $1.57 \pm 0.35$ & $1.59 \pm 0.45$ \\
\hline Glomeruli with crescents (\%) & $0.2 \pm 0.4$ & $0.0 \pm 0.0$ & $8.1 \pm 2.2$ & $3.3 \pm 2.6 \mathrm{C}$ & $8.0 \pm 4.6$ & $5.1 \pm 2.7$ \\
\hline Autologous IgG end point titer (glomerulus) & $1: 1,600$ & $1: 800$ & $1: 1,600$ & $1: 1,600$ & $1: 1,600$ & $1: 1,600$ \\
\hline Complement C3 deposition (glomerulus) & $50.8 \pm 15.0$ & $22.1 \pm 7.4 \mathrm{C}$ & $20.5 \pm 6.5$ & $20.4 \pm 8.2$ & n.d. & n.d. \\
\hline $\mathrm{CD} 3^{+} \mathrm{T}$ cells (glomerulus) & $0.19 \pm 0.03$ & $0.23 \pm 0.06$ & $0.42 \pm 0.13$ & $1.61 \pm 0.72^{B}$ & $0.26 \pm 0.10$ & $1.71 \pm 0.28^{\mathrm{D}}$ \\
\hline $\mathrm{CD}^{+} \mathrm{T}$ cells/HPF (interstitial) & $10.7 \pm 2.0$ & $13.1 \pm 1.9$ & $42.4 \pm 11.8$ & $81.7 \pm 24.8^{B}$ & $48.0 \pm 1.8$ & $142.1 \pm 13.3^{\mathrm{D}}$ \\
\hline ER-HR3+ macrophages (glomerulus) & $0.07 \pm 0.02$ & $0.02 \pm 0.010$ & $0.13 \pm 0.02$ & $0.11 \pm 0.02$ & $0.10 \pm 0.02$ & $0.11 \pm 0.02$ \\
\hline ER-HR3+ macrophages/HPF (interstitial) & $1.3 \pm 0.3$ & $0.3 \pm 0.2^{D}$ & $24.1 \pm 4.2$ & $22.7 \pm 5.5$ & $28.4 \pm 9.7$ & $30.5 \pm 11.7$ \\
\hline
\end{tabular}

AValues are mean $\pm \mathrm{SD} ; n=4-7$ per group. ${ }^{\mathrm{B}} P<0.05,{ }^{\mathrm{C}} P<0.01$, and $\mathrm{D} P<0.001$ compared with wild-type mice. n.d., not done.

TNFR2 expression on $\alpha \beta T$ cells is not required for induction of GN. Our previous study showed that $\mathrm{T}$ cells are required for the induction of nephrotoxic nephritis (34), and this was confirmed with the antiserum used in the current study. To investigate whether TNFR2 expressed on $\alpha \beta$ T cells was required for GN, we reconstituted $\alpha \beta$ T cell-deficient mice with either wild-type or TNFR2deficient T cells and subjected them to nephrotoxic nephritis. Both groups of mice developed GN to a similar extent. (Figure 9, A and $\mathrm{B}$ and Table 3 ). $\alpha \beta \mathrm{T}$ cell-deficient mice reconstituted with T cells prepared from $\alpha \beta$ T cell-deficient mice did not develop GN or leukocyte infiltrates (Figure 9, A-C). Thus, despite the reliance of this model on $\alpha \beta$ T cells and TNFR2, and the prominent expression of this receptor on T cells, TNFR2 on these cells is not required for the progression of GN.

TNFR2 expression by intrinsic cells is required for the development of $G N$. To determine the extent to which intrinsic cell- versus leukocyteexpressed TNFR2 is required for disease development, we analyzed mice expressing TNFR2 only in BM-derived cells or intrinsic cells that were generated by BM transplantation between wild-type and TNFR2-deficient animals. The successful generation of the appropriate chimeras was analyzed by flow cytometry of the congenic markers CD45.1 and CD45.2 expressed on wild-type and TNFR2deficient leukocytes, respectively. To control for any effect of radiation or BM transplantation on kidney function, we also generated chimeric mice with CD45.1 congenic wild-type BM transplanted into CD45.2 positive wild-type recipients.

The BM chimeras were subjected to nephrotoxic nephritis. Chimeric mice with TNFR2-deficient BM and intact renal TNFR2 expression (TNFR2 $2^{-/-} \rightarrow$ WT chimeras) developed albuminuria that was comparable to the control chimeras with TNFR2-positive BM and intrinsic renal cells (WT $\rightarrow$ WT chimeras) (Figure 10A). Consistent with these findings, both groups of mice had hypoalbuminemia and hypercholesterolemia (Figure 10B). In contrast, mice with TNFR2-positive BM-derived cells but TNFR2-deficient renal cells (WT $\rightarrow$ TNFR2 ${ }^{-/-}$chimeras) developed minimal proteinuria (Figure 10A) and had normal serum values (Figure 10B). Despite the divergence of overall phenotype in the 2 groups of chimeras, both TNFR2 $2^{-/-} \rightarrow$ WT chimeras and WT $\rightarrow$ TNFR2 $2^{-/-}$chimeras had significantly reduced serum creatinine values compared with WT $\rightarrow$ WT control chimeras at day 21 (Figure 10). The difference in data obtained from proteinuria and serum creatinine measurements may result from the fact that proteinuria is a much more sensitive indicator of glomerular injury than serum creatinine. A higher threshold of renal injury may be required to elevate serum creatinine, and TNFR2 $2^{-\prime} \rightarrow$ WT chimeras may fail to reach this threshold. Indeed, analysis of renal histology suggested that the severe glomerular injury in WT $\rightarrow$ WT chimeras at day 21, as indicated by significant glomerular PAS deposition and crescent formation, was attenuated in TNFR2 $2^{-\prime} \rightarrow$ WT chimeras. Notably, glomerular damage was nearly absent in WT $\rightarrow$ TNFR2 $2^{-/}$chimeras (Table 4). Glomerular deposition of autologous mouse IgG was similar in all 3 chimeras (Table 4), which is consistent with results obtained in wild-type and TNFR2-deficient mice (Table 1). However, glomerular deposition of complement C3 was reduced in WT $\rightarrow$ TNFR2 $2^{-/}$chimeras compared with TNFR2 $2^{-1-} \rightarrow$ WT and $\mathrm{WT} \rightarrow \mathrm{WT}$ chimeras (Table 4). In TNFR2 $2^{--} \rightarrow$ WT chimeras, the number of infiltrating renal $\alpha \beta \mathrm{T}$ cells, but not macrophages, was reduced compared with that in WT $\rightarrow$ WT chimeras. This may have contributed to the preservation of some renal function, as serum creatinine levels in these mice were not elevated despite substantial proteinuria and glomerular injury. Strikingly, WT $\rightarrow$ TNFR2 ${ }^{-/}$chimeras exhibited minimal renal $\alpha \beta \mathrm{T}$ cell and macrophage infiltration, which is consistent with the absence of significant proteinuria and glomerular injury in these mice (Figure 10C). Taken together, these data demonstrate that TNFR2 expression

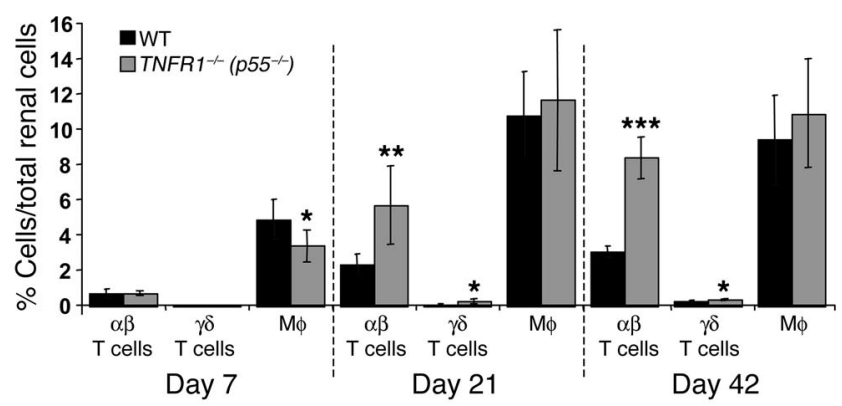

Figure 6

Renal leukocyte infiltrates in nephritic wild-type and TNFR1-deficient mice. Flow cytometry of $\alpha \beta$ T cells, $\gamma \delta$ T cells, and F4/80-positive macrophages in nephritic kidneys harvested at the indicated days after induction of nephrotoxic nephritis ( $n=7-9$ per group). Data represent the mean \pm SD. ${ }^{*} P<0.05,{ }^{* *} P<0.01,{ }^{* \star *} P<0.001$ compared with wild-type. $\mathrm{M} \phi$, macrophage. 
A
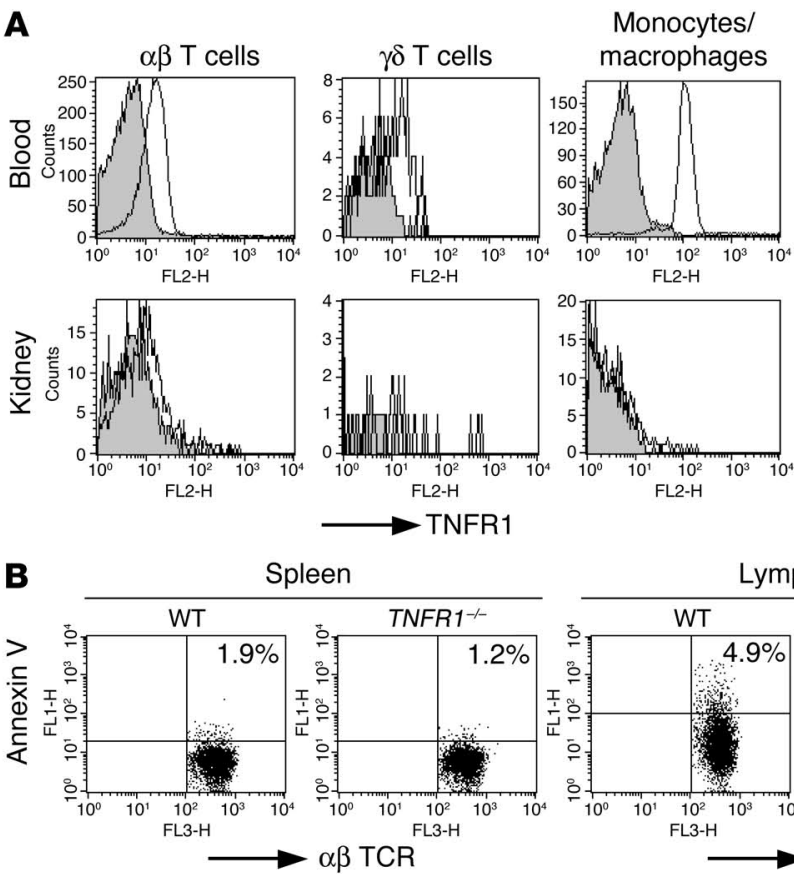

Spleen
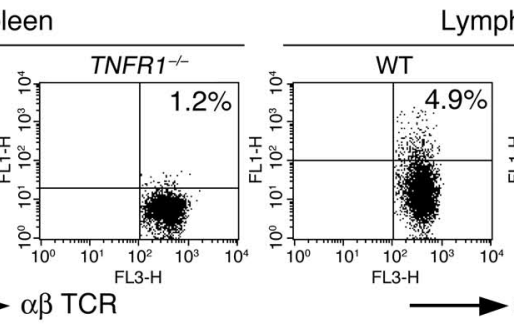

Lymph node
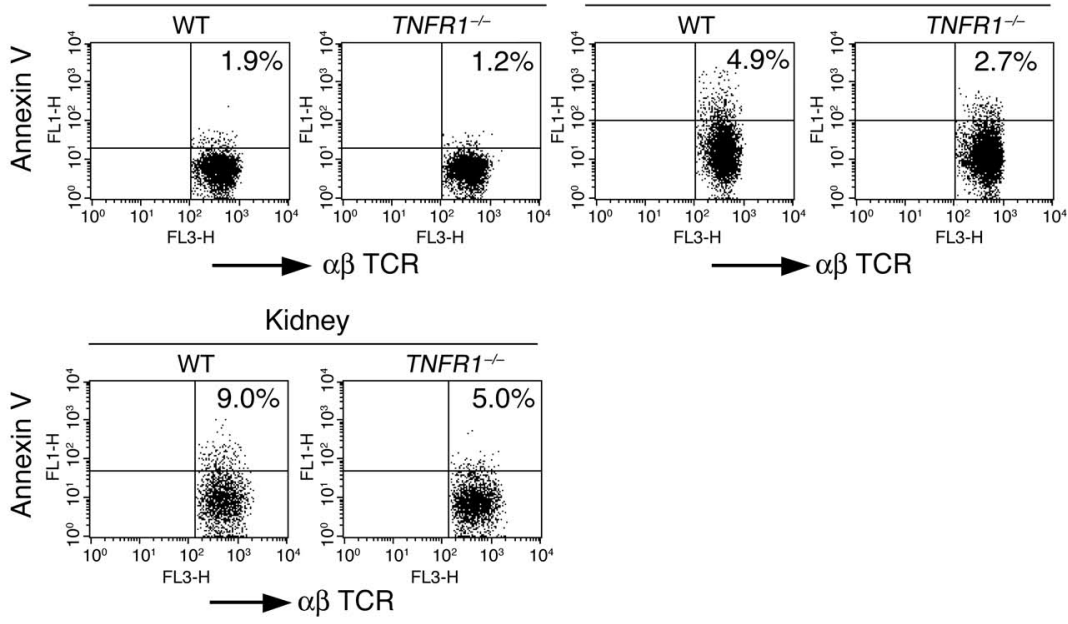

C
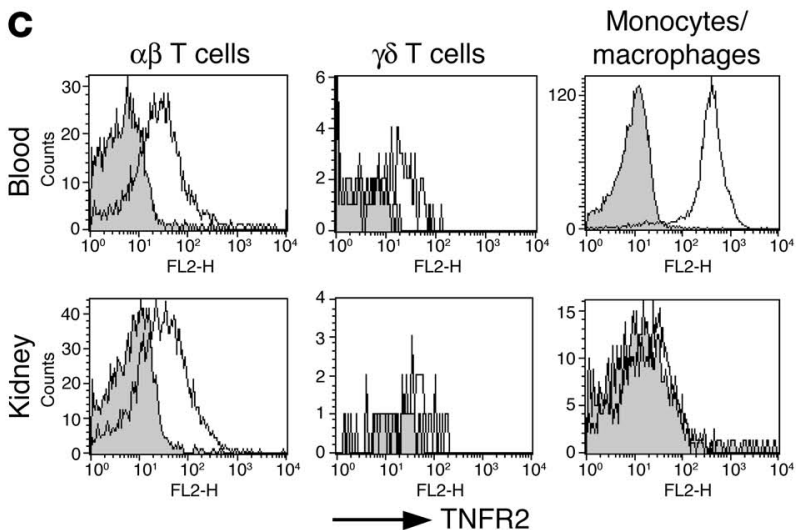

on leukocytes partially contributes to disease progression, whereas TNFR2-expressing intrinsic cells are essential for the development of GN. The disease-protective effect in TNFR2-deficient mice is likely caused by the lack of TNFR2 expression on parenchymal renal cells, such as glomerular endothelial cells, although it is also possible that the lack of TNFR2 expression on intrinsic cells other than renal cells inhibits disease development.

\section{Discussion}

TNF is an important mediator of chronic inflammatory immunemediated disorders and plays an essential role in the pathogenesis of experimental GN $(4-10,17)$. In this study, we have defined

\section{Figure 7}

Flow cytometric analysis of peripheral and renal leukocyte subsets isolated from nephritic mice. Whole-blood and single-cell suspensions obtained from spleen and nephritic kidneys of wild-type mice at day 21 were stained for (A) TNFR1 or (C) TNFR2 and $\mathrm{CD} 3 \varepsilon, \alpha \beta$ TCR, and $\gamma \delta$ TCR or CD $3 \varepsilon$ and F4/80. After gating on $\mathrm{CD}^{+} \alpha \beta \mathrm{TCR}^{+}, \mathrm{CD}^{+} \gamma \delta \mathrm{TCR}^{+}$, and CD3- $\mathrm{F} 4 / 80^{+}$cells, the fraction of TNFR-positive cells was determined in comparison to staining with the isotype control antibody (Table 3). Representative histograms for staining of each cell population in the peripheral blood and kidney are shown in comparison to those for staining of the isotype control antibody (shaded histograms). (B) The proportion of apoptotic $\alpha \beta$ T cells in spleen, lymph nodes, and kidneys of wild-type and TNFR1-deficient mice was determined after staining for annexin $\mathrm{V}, \mathrm{CD} 3 \varepsilon$, and $\alpha \beta$ TCR. Dead cells were excluded after propidium iodide staining. Representative dot plots of 2 independent experiments are shown, and the fraction of apoptotic $\alpha \beta$ T cells is indicated in the upper-right of the plots.

differential roles for the 2 TNFRs, TNFR1 and TNFR2, in the development of accelerated nephrotoxic nephritis, a model of progressive immune complex-mediated GN dependent on complement and $\alpha \beta$ and $\gamma \delta$ T cells $(33,34)$. TNFR1 has an immunosuppressive role likely through regulation of the humoral response and $\mathrm{T}$ cell apoptosis/accumulation, the latter being dependent on the expression of TNFR1 on cells other than the $T$ cell itself. In sharp contrast, TNFR2 is induced on the glomerular endothelium of the nephritic kidney and its expression is required on intrinsic cells for complement deposition and the initiation and progression of GN.

The nearly complete protection of TNFR2deficient mice in GN suggests that TNFR2 plays a nonredundant role in mediating renal injury, and this finding is the first demonstration to our knowledge of an essential proinflammatory function of TNFR2 in glomerular disease. TNFR2-deficient mice have normal spleen and lymph node architecture, and the germinal center reaction and formation of follicular dendritic cell networks are intact (26). Delayed-type hypersensitivity responses in TNFR2-deficient mice are comparable to those in wild-type mice (38). Furthermore, TNFR2-deficient mice mounted a systemic immune response toward the nephrotoxic antibody, and the reconstitution studies in $\alpha \beta$ T cell-deficient mice suggest that TNFR2-deficient T cells are competent to induce GN. Together, these data demonstrate that humoral and cell-mediated immunity are intact in mice lacking TNFR2. Thus, the observed protection in TNFR2-deficient mice subjected to GN suggests that TNFR2 is required for the effector phase of nephrotoxic nephritis.

TNFR2 is expressed on monocytes/macrophages and on peripheral T cells in blood and lymphoid organs. It is also present on infiltrating renal $\mathrm{T}$ cells during $\mathrm{GN}$ but is absent on renal macrophages. The latter observation suggests that macrophage- 


\section{Table 3}

TNFR1 and TNFR2 expression on leukocytes in the peripheral blood, spleen, and nephritic kidney at day 21 after induction of nephrotoxic serum nephritis ${ }^{A}$

$\begin{array}{lccc}\text { \% Positive cells } & & \text { TNFR1 } & \text { TNFR2 } \\ \alpha \beta \text { T cells } & \text { Blood } & 28.7 \pm 8.4 & 37.5 \pm 14.2 \\ \alpha \beta \text { T cells } & \text { Spleen } & 22.4 \pm 6.6 & 43.5 \pm 16.5 \\ \alpha \beta \text { T cells } & \text { Kidney } & 10.8 \pm 7.8^{\mathrm{B}} & 49.3 \pm 14.0 \\ \gamma \delta \text { T cells } & \text { Blood } & 32.4 \pm 13.9 & 55.6 \pm 13.4 \\ \gamma \delta \text { T cells } & \text { Spleen } & 45.1 \pm 2.7 & 54.4 \pm 17.8 \\ \gamma \delta \text { T cells } & \text { Kidney } & 7.8 \pm 5.0^{\mathrm{B}} & 21.5 \pm 16.0^{\mathrm{B}} \\ \text { F4/80+ monocytes } & \text { Blood } & 92.7 \pm 4.0 & 94.6 \pm 4.9 \\ \text { F4/80+ macrophages } & \text { Spleen } & 84.5 \pm 5.3^{\mathrm{B}} & 95.0 \pm 0.3 \\ \text { F4/80+ macrophages } & \text { Kidney } & 1.2 \pm 1.7^{\mathrm{C}} & 0.1 \pm 0.1^{\mathrm{C}}\end{array}$

ATNFR surface expression was analyzed in wild-type mice by flow cytometry. Values are mean $\pm \mathrm{SD} ; n=4-7$ per group. ${ }^{\mathrm{B} P}<0.05$, ${ }^{C} P<0.001$, compared with blood.

expressed TNFR2 in the kidney is unlikely to mediate local renal injury. Although it was hypothesized that recruitment of activated macrophages into the kidney may require TNFR2 expression on their surface, this was also not essential, since macrophage accumulation was not significantly reduced in BM chimeras deficient in TNFR2 in circulating cells. TNFR2 expression on T cells was also not essential for disease progression.

TNFR2 expression was induced in intrinsic renal cells of nephritic kidneys, and confocal microscopy revealed renal TNFR2 protein expression localized to glomerular endothelial cells. In addition, endothelial cell-expressed TNFR2 was present in postcapillary venules of the cortical interstitium. Thus, renal TNFR2 expression localized both with the site of the primary glomerular damage and the site of secondary interstitial nephritis that accompanies the glomerular lesion. Indeed, our findings are consistent with the previously reported glomerular expression of TNFR2 in human glomerulopathies (24). The observed pattern of TNFR2 expression was highly specific to the disease process. TNFR2 protein was not detected in normal mouse kidney. Moreover, TNFR2 expression was restricted to tubular epithelial cells and was undetectable in glomeruli or interstitial venules in mice with tubular damage resulting from obstructive nephropathy. Importantly, analyses of GN in mice chimeric for TNFR2 expression indicate that TNFR2 expression on intrinsic cells, likely glomerular endothelial cells, is critical for the development of all parameters of GN. However, our data cannot rule out a possible role for intrinsic cells other than renal cells (e.g., activation of complement by extrarenal endothelial cells) in mediating GN. In contrast, leukocyteexpressed TNFR 2 only partly contributes to renal injury.

How does renal cell-expressed TNFR2 mediate proteinuria and glomerular damage? One mechanism is the promotion of complement deposition, as suggested by our studies, in which a marked attenuation of glomerular complement deposition was observed in both TNFR2-deficient mice and mice lacking TNFR2 expression on intrinsic cells. We propose that endothelial TNFR2 could contribute to complement activation/deposition. Interestingly, endothelial cells activated by TNF promote complement deposition in areas of exposed subendothelial matrix in vitro, which is initiated by cytokine-induced retraction of these cells (39). TNFR2 could be involved in this process. Complement fixation on exposed subendothelial basement membrane in vivo promotes glomerular injury and inflammation $(35,36)$. Moreover, complement is important in the development of proteinuria in our model of accelerated nephrotoxic nephritis, as C5-deficient mice developed minimal albuminuria at days 14 and 21 (33). Complement-mediated injury may occur through the generation of deleterious terminal pathway activation products and/or split products, such as $\mathrm{C} 3 \mathrm{a}$, which modulate proinflammatory cytokine expression in monocytes and leukocyte activation and recruitment (39). It is also possible that TNFdependent upregulation of vascular adhesion molecules $(18,19)$, which would facilitate the infiltration of effector leukocytes into the kidney, is TNFR2 mediated. Nevertheless, it is noteworthy that we detected no differences in renal ICAM-1 expression in nephritic wild-type and TNFR2-deficient mice by immunohistochemistry, and positive signals for E-selectin were not detected in either group of mice at the time points tested (data not shown).

Most classic proinflammatory effects of TNF, including the release of other inflammatory cytokines and the upregulation of vascular adhesion molecules, are mediated through TNFR1 signaling $(21,25,26)$. In our studies, TNFR1-deficient mice exhibited only a delay in the development of GN but were not protected from disease. This was associated with a significant decrease in circulating autologous antibody against rabbit IgG, although its glomerular deposition was equivalent to that observed in wildtype mice. The reduction in autologous antibodies indicates a defect in the systemic immune response toward the injected rab-
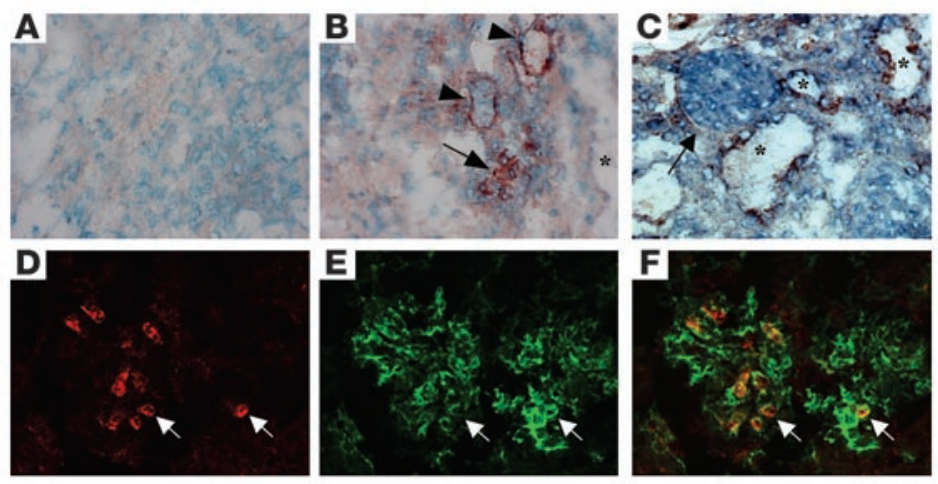

\section{Figure 8}

Localization of TNFR2 in nephritic kidneys. Tissue sections were processed for immunohistochemistry (A-C). (A) TNFR2 expression was not present in the renal cortex of normal wild-type mice without nephritis. (B) Expression of TNFR2 was detected in glomeruli (arrow) and postcapillary venules of the renal cortex (arrowheads) of wild-type mice subjected to GN. Staining was not detected in kidneys of TNFR2-l- nephritic mice (data not shown), which demonstrated specificity of the antibody for TNFR2. (C) TNFR2 was present in epithelial cells of tubules $\left(^{*}\right)$ but was not present in the glomerulus (arrow) of wild-type mice subjected to unilateral ureteral ligation, which results in epithelial cell damage. Original magnification $(\mathbf{A}-\mathbf{C}), \times 630$. Tissue sections of a kidney from a wild-type mouse subjected to GN were processed with fluorophore-conjugated antibody to TNFR2 (red) and CD31/PECAM-1 (green) and imaged by confocal microscopy (D-F). D depicts a single $0.5-\mu \mathrm{m}$ confocal slice showing TNFR2 (red) enriched in glomerular capillaries (arrows) as costained (E) by PECAM-1 (green). (F) Colocalization of TNFR2 and the endothelial cell marker PECAM-1 (yellow) are shown in the merged image. Original magnification (D-F), $\times 600$. 

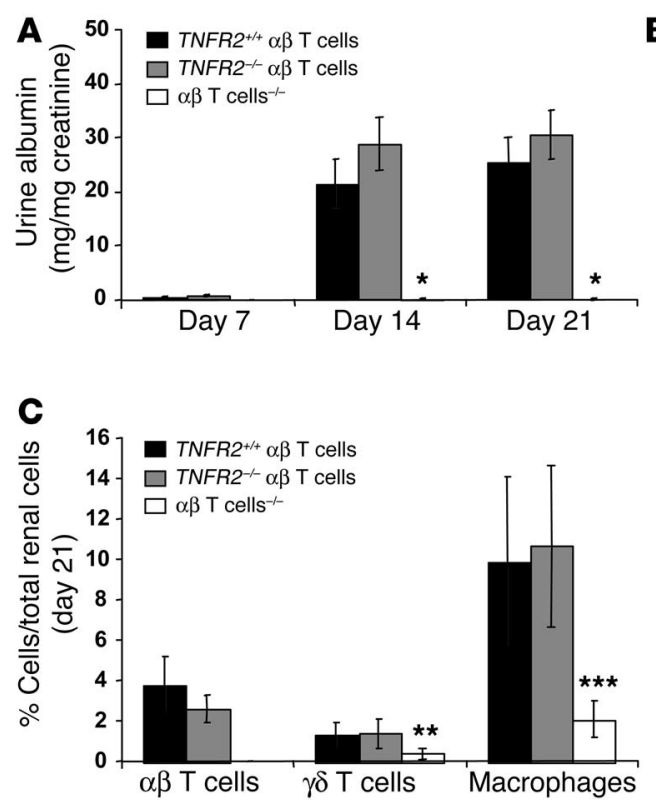

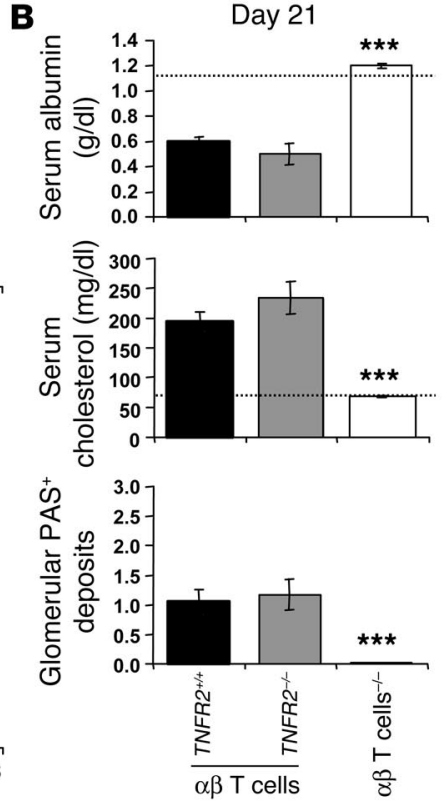

\section{Figure 9}

TNFR2-deficient $\alpha \beta$ T cells induce GN. $\alpha \beta$ T cell-deficient mice reconstituted with splenic $T$ cell preparations from wild-type, TNFR2-deficient, or $\alpha \beta$ T celldeficient mice were subjected to nephrotoxic serum nephritis. (A) Mice with TNFR2-deficient $\alpha \beta$ T cells developed albuminuria similarly to mice reconstituted with wild-type $\alpha \beta$ T cells, whereas $\alpha \beta$ T cell-deficient control mice that were adoptively transferred with splenic T cell preparations lacking $\alpha \beta$ T cells did not develop albuminuria. Data represent the mean \pm SEM ( $n=6$ per group). (B) Hypoalbuminemia, hypercholesterolemia, and glomerular PAS deposits developed in mice with wild-type and TNFR2-deficient $\alpha \beta$ T cells but not in $\alpha \beta$ T cell-deficient control mice. Data represent the mean \pm SEM ( $n=6$ per group). (C) Renal leukocyte infiltration was similar in mice with wild-type and TNFR2-deficient $\alpha \beta$ T cells but absent in $\alpha \beta$ T cell-deficient control mice. Data represent the mean \pm SD ( $n=6$ per group). The dotted lines indicate baseline values observed in normal wild-type mice. ${ }^{*} P<0.05,{ }^{* *} P<0.01$, and ${ }^{* * *} P<0.001 \mathrm{com}$ pared with mice with wild-type $\alpha \beta$ T cells. bit anti-GBM antibody. The reduction in circulating autologous antibodies in TNFR1-deficient mice is not responsible for the protection observed in these mice at early time points, since mice lacking B cells and autologous antibody production ( $\mu$ chain-deficient mice) have proteinuria similar to that of wild-type mice at days 7 and 14 (ref. 34 and data not shown for the serum used in the current experiments). Thus, we propose that the early protection from glomerular injury as well as the decreased humoral response may result from an impaired generation of sensitized $\mathrm{T}$ cells in TNFR1-deficient mice. Sensitized T cells not only provide B cell help for the production of autologous antibodies in accelerated nephrotoxic nephritis but are required for the development of GN independently of antibody production (34). Indeed, TNFR1 is required for $\mathrm{T}$ cell priming after subcutaneous injection of antigen (20), and delayed-type hypersensitivity responses are impaired in TNFR1-deficient mice (38). Finally, TNFR1-deficient mice, like TNF-deficient animals, are defective in the formation of germinal centers and follicular dendritic cell networks in secondary lymphoid organs $(21,26,40)$. Taken together, these data suggest that an impaired adaptive immune response

\section{Figure 10}

TNFR2 expression by intrinsic renal cells is required for the development of GN. (A) Albuminuria was evaluated in chimeric mice with TNFR2-deficient BM (TNFR2 ${ }^{-l-} \rightarrow$ WT chimeras) or TNFR2-deficient renal cells (WT $\rightarrow$ TNFR2 ${ }^{-1-}$ chimeras) at days 7,14 , and 21 after injection of nephrotoxic serum. Wild-type mice transplanted with wild-type BM (WT $\rightarrow$ WT chimeras) served as controls ( $n=7-11$ per group). Data represent the mean \pm SEM. (B) Serum levels for albumin, cholesterol, and creatinine were determined at day 21 ( $n=7-11$ per group). Data are mean \pm SEM. (C) Renal leukocyte infiltrates in BM chimeric mice at days 7,21 , and 42 after injection of nephrotoxic serum ( $n=6-11$ per group). Data represent the mean \pm SD. ${ }^{*} P<0.05$, ${ }^{* *} P<0.01$, and ${ }^{* \star \star} P<0.001$ compared with control chimeras. in TNFR1-deficient mice is responsible for the delay in onset of nephritis observed in these animals.

Despite the initial delay in the development of nephritis, at later time points, TNFR1-deficient mice exhibited disease that was largely comparable to that in wild-type mice. This may be attributed to excessive accumulation of $\mathrm{T}$ cells in these mice. Fewer apoptotic $\mathrm{T}$ cells were observed in nephritic TNFR1-deficient mice in all organs investigated, and this reduction in apoptosis may account for the increased size of draining paraaortic lymph nodes (data not shown) and the excessive accumulation of renal $\mathrm{T}$ cells in nephritic mice. Thus, TNFR1-mediated apoptosis of activated T cells may be a mechanism to control renal $\mathrm{T}$ cell responses in $\mathrm{GN}$ as reported in a study of lupus-prone C57BL/6-lpr/lpr mice lacking TNFR1 (41). Despite the increase in glomerular and interstitial $\mathrm{T}$ cell infiltrates
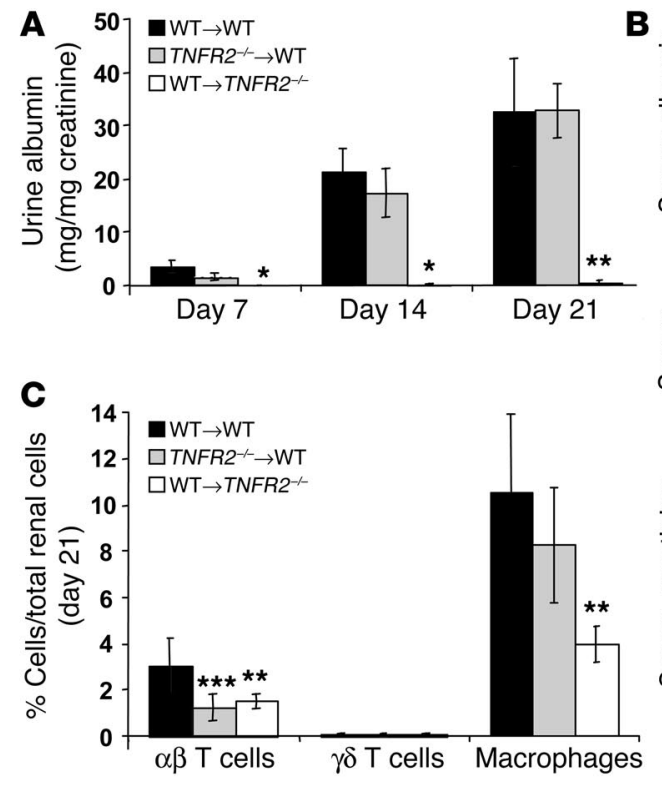


\section{Table 4}

Comparison of glomerular injury, glomerular deposition of autologous Ig $\mathrm{G}$ and glomerular complement deposition in WT $\rightarrow$ WT, TNFR2 $2^{--} \rightarrow \mathrm{WT}$, and WT $\rightarrow$ TNFR2 $2^{--}$chimeras

$\begin{array}{lccc}\text { Day 21 } & \text { WT } \rightarrow \text { WT } & \text { TNFR2 }- \text { - } \rightarrow \text { WT } & \text { WT } \rightarrow \text { TNFR2 } \\ \text { PAS deposition (glomerulus) } & 1.50 \pm 0.02 & 1.00 \pm 0.39^{A} & 0.10 \pm 0.05^{B} \\ \text { Glomeruli with crescents (\%) } & 5.2 \pm 3.4 & 1.3 \pm 1.7 & 0.18 \pm 0.05^{C} \\ \text { Autologous IgG end point titer (glomerulus) } & 1: 1,600 & 1: 1,600 & 1: 1,600 \\ \text { Complement C3 deposition (glomerulus) } & 29.3 \pm 7.4 & 30.3 \pm 7.7 & 6.6 \pm 3.6^{\mathrm{B}}\end{array}$

Values are mean $\pm \mathrm{SD}, n=9$ per group. ${ }^{A} P<0.01,{ }^{\mathrm{B}} P<0.001$, and ${ }^{\mathrm{C}} P<0.05$, compared with wildtype mice.

in TNFR1-deficient mice, parameters of glomerular injury were not significantly worse compared with those in wild-type mice. As TNFR1 mediates both proinflammatory ( $\mathrm{T}$ cell priming) and immunosuppressive (apoptosis of activated T cells) responses during nephrotoxic nephritis, the phenotype in TNFR1-deficient mice may reflect the net effect of both $\mathrm{T}$ cell-dependent functions. Moreover, it is noteworthy that the extent of renal macrophage but not $\mathrm{T}$ cell accumulation correlated with the degree of glomerular injury in wild-type and TNFR1-deficient mice at all time points studied.

Although TNFR1-mediated apoptosis of activated T cells has been described (22), the adoptive T cell transfer studies suggest that neither the delayed onset of disease nor the excessive renal $\mathrm{T}$ cell accumulation and reduced apoptosis of $\alpha \beta \mathrm{T}$ cells at later time points is dependent on TNFR 1 on $\alpha \beta$ T cells. We propose that TNFR1-mediated differentiation and activation of APCs (40) might facilitate the rapid generation of sensitized $T$ cells that are required for disease induction in our model. Apoptosis of T cells during presentation of antigen can be induced by APCs expressing Fas ligand $(42,43)$, and we speculate that TNFR1 expressed on APCs such as glomerular mesangial and tubular epithelial cells may have a functional role in this process $(15,44)$. Mesangial cells upregulate Fas ligand upon TNF stimulation in vitro (45). Fas ligand is also expressed in tubular epithelium and in injured glomeruli during immune complex nephritis (46) and induces apoptosis in Fas-sensitive lymphoid cell lines in vitro (46).

In summary, TNFR2 expressed on intrinsic cells, likely glomerular endothelial cells, specifically mediated organ-specific tissue damage in GN, independent of TNFR1. In contrast, TNFR1 deficiency was not protective throughout disease development and actually resulted in increased disease indices such as the accumulation of renal $T$ cells in our model. The successful development and increasing clinical use of anti-TNF therapies for the treatment of chronic autoimmune disease demonstrate that neutralization of endogenous TNF can control progression of disease in humans. However, anti-TNF therapies in inflammatory immune-mediated disorders such as GN may lead to unpredictable outcomes, due to the contrasting proinflammatory and immunosuppressive functions of TNF in these conditions $(20,21)$. Indeed, anti-TNF therapies, although beneficial for the majority of patients with rheumatoid arthritis or inflammatory bowel disease, has led to the formation of anti-DNA antibodies and the development of lupus-like syndromes in some patients. Moreover, TNF blockade in multiple sclerosis has frequently caused immune activation and disease exacerbation (21). Our studies indicate that specific blockade of TNFR2 may be preferable to anti-TNF treatments in human GN, especially in the context of autoimmune disease such as systemic lupus erythematodes. Specific TNFR2 antagonism would preserve immunosup- pressive effects of TNF such as apoptosis of autoreactive T cells (21) but block TNF dependent proinflammatory functions that lead to glomerular dysfunction. Thus, therapeutic blockade of TNFR2 may be a promising strategy in the treatment of human GN.

\section{Methods}

Mice. Mouse strains with targeted deletion of TNFR1 (TNFR1-/-, B6.129-Tnfrsf1 $a^{\text {tmIMak }}$ ) (47), TNFR2 (TNFR2 $2^{--}$, B6.129-Tnfrsf1 $1 b^{\text {tmIMwm }}$ ) (48), and their wild-type C57BL/6J controls were obtained from The Jackson Laboratory. TNFR1 $1^{-/}$and TNFR2 $2^{-/}$mice were backcrossed 13 and 10 generations to C57BL/6J mice, respectively. $\alpha \beta \mathrm{T}$ cell-deficient mice (B6.129-Torb ${ }^{\text {tm } 1 M o m}$ ) (49) and CD45.1 congenic mice (B6.SJL-Ptprc ${ }^{a} P e 3^{b} /$ BoyJ) (The Jackson Laboratory) were also obtained on a C57BL/ 6 background. Animals were maintained in a virus- and antibody-free facility at the Braunwald Medical Research Center (Brigham and Women's Hospital). Experiments were approved by the Harvard Medical School animal care and use committee.

Adoptive T cell transfer. We purified CD90-positive T cells from spleens after erythrocyte lysis using a MACS CD90 MicroBeads kit (Miltenyi Biotec). This protocol typically yielded more than $95 \% \mathrm{~T}$ cells, as assessed by flow cytometry. A total of $3 \times 10^{6}$ splenic T cells from wild-type, $\alpha \beta \mathrm{T}$ cell-deficient, TNFR1-deficient, or TNFR2-deficient mice were adoptively transferred into $\alpha \beta \mathrm{T}$ cell-deficient mice by tail vein injection. Efficiency of the adoptive transfer in each experimental mouse was demonstrated by flow cytometry of blood, spleen, and lymph node cells obtained at the end of the experiment.

$B M$ transplantation. We generated TNFR2 BM chimeric mice by transplanting TNFR2-deficient BM into lethally irradiated wild-type recipients (TNFR2 $2^{--} \rightarrow$ WT chimeras) or vice versa (WT $\rightarrow$ TNFR2 $2^{-/}$chimeras). To assess the efficiency of BM replacement in each experimental group, we used CD45.1 congenic C57BL/6J wild-type mice as donors or recipients, respectively, so as to distinguish CD45.2-positive TNFR2-/- leukocytes from CD45.1-positive wild-type leukocytes by flow cytometry. Irradiated CD45.2-positive wild-type recipients reconstituted with CD45.1 congenic wild-type BM were used as controls (WT $\rightarrow$ WT chimeras). Irradiation and $\mathrm{BM}$ reconstitution was carried out as described previously (33). Circulating leukocyte numbers and leukocyte subpopulations at 6 weeks after transplantation were normal as assessed by flow cytometry. Moreover, flow cytometric analysis of peripheral blood samples obtained 6 weeks after transplantation, as well as peripheral blood, spleens, and kidneys harvested at the end of each experiment, revealed that more than $95 \%$ of leukocytes in all 3 types of chimeras were of donor origin (data not shown).

Nephrotoxic nephritis. Nephrotoxic serum was prepared by immunizing rabbits with mouse GBM, as described by Schrijver et al. (50). Rabbit immunization and serum collection were conducted by Covance Research Products Inc. Accelerated nephrotoxic serum nephritis was induced in male mice between 7 and 9 weeks essentially as previously reported $(33,34)$. BM chimeric mice were used at 14 weeks of age, 6 weeks after BM transplantation. Adoptive $\mathrm{T}$ cell transfers were performed 2 days before preimmunization. Briefly, mice were preimmunized subcutaneously in the right foot pad with $0.1 \mathrm{mg}$ rabbit IgG (Jackson ImmunoResearch Laboratories Inc.) in Freund incomplete adjuvant and nonviable desiccated Mycobacterium tuberculosis $\mathrm{H} 37 \mathrm{Ra}$ (BD Biosciences - Pharmingen). Three days later, mice were injected intravenously with $100 \mu \mathrm{l}$ heat-inactivated, filter-sterilized nephrotoxic serum. Spot urine samples were collected at weekly intervals. At selected time points (day 7, 21, or 42 after injection of nephrotoxic 
serum), mice were anesthetized with methoxyflurane, and peripheral blood was collected by retroorbital bleeding into glass tubes to obtain serum. The animals were then euthanized by $\mathrm{CO}_{2}$ inhalation, and both kidneys were harvested for histologic analysis and flow cytometry.

Humoral immune response to rabbit antiserum. Circulating mouse anti-rabbit immunoglobulin antibodies were measured by ELISA on serum collected at the end of each experiment as previously described (33).

Functional assessment of renal injury. Urine albumin concentrations and urinary creatine levels were determined by ELISA and alkaline picric acid method as previously described (33). Albuminuria was expressed as milligrams albumin per milligrams urinary creatinine to standardize urine albumin excretion for glomerular filtration rate and urinary concentration. Serum values for creatinine, albumin, and cholesterol were obtained with an autoanalyzer (Hitachi 917; Roche Diagnostics Corp.) at The Children's Hospital, Boston, Massachusetts, USA.

Histologic assessment of renal injury. We stained $4-\mu \mathrm{m}$ coronal sections of paraffin-embedded kidneys with PAS reagent for analysis of glomerular injury. The presence of PAS-positive deposits within glomeruli was graded semiquantitatively in 100 equatorial glomerular cross-sections per mouse as previously described (34). Glomeruli were considered to exhibit crescent formation when 3 or more layers of cells were observed in the Bowman space.

Immunofluorescence microscopy was performed on $4-\mu \mathrm{m}$ frozen sections. Semiquantitative assessment of the autologous (mouse) glomerular IgG deposition was performed by determining the end point positive titer for detection of staining using serial dilutions of the FITC-conjugated goat anti-mouse IgG $(1.5 \mathrm{mg} / \mathrm{ml}$, absorbed against rabbit; Jackson ImmunoResearch Laboratories Inc.). Deposition of complement C3 was visualized with a FITC-conjugated goat $\mathrm{F}\left(\mathrm{ab}^{\prime}\right)_{2}$ fragment against mouse complement C3 (4 mg/ml; ICN Pharmaceuticals) at a dilution of 1:300. C3 deposition was quantitated by image analysis. Sections were examined with a fluorescent microscope (Nikon Eclipse TE2000-U; Nikon Instruments) using a $20 \times$ lens. Images of 10 glomeruli per mouse were captured with a charge coupled device camera and exported to Adobe Photoshop 6.0 software (Adobe Systems Inc.), and glomerular fluorescence intensity (mean luminosity) was measured. Staining with a FITC-labeled goat $\mathrm{F}\left(\mathrm{ab}^{\prime}\right)_{2}$ fragment served as negative control. All morphometric assessments were performed in a blinded protocol.

Immunohistochemistry. Infiltrating renal leukocytes and apoptotic cells were detected using a 3-layer immunoperoxidase technique. We incubated 4- $\mu \mathrm{m}$ paraffin-embedded sections with primary antibodies to stain for $\mathrm{T}$ cells (cross-reactive rat anti-human CD3, clone CD3-12, $1 \mathrm{mg} / \mathrm{ml}, 1: 100$; Serotec), macrophages (rat anti-mouse ER-HR3 [ref. 51], $0.6 \mathrm{mg} / \mathrm{ml}, 1: 50$; BMA Biomedicals), and apoptotic cells (rabbit anti-cleaved caspase 3 , clone Asp175, 1:50; Cell Signaling Technology). In order to evaluate the number of glomerular and interstitial leukocytes and apoptotic cells, we counted positively stained cells in 100 glomeruli or in 10 cortical HPFs (magnification, $\times 400$ ) per mouse, respectively.

To detect renal TNFR2 expression, we stained 4- $\mu \mathrm{m}$ cryostat-cut, acetone-fixed sections with a polyclonal goat anti-murine TNFR2 antibody $(0.2 \mathrm{mg} / \mathrm{ml}$, diluted 1:200; Santa Cruz Biotechnology Inc.). Biotin-conjugated rat anti-goat antibody was used as the secondary antibody. The use of goat IgG as the primary antibody served as a negative control. In addition, negative staining of tissue sections from TNFR2-deficient mice demonstrated the specificity of the TNFR2 antiserum. To identify the resident renal cell type expressing TNFR2, we prepared samples for confocal microscopy as follows: $5-\mu \mathrm{m}$ frozen tissue sections were fixed in acetone/methanol for 2 minutes at $4^{\circ} \mathrm{C}$, washed, and incubated with Alexa 488-conjugated hamster anti-mouse PECAM (clone 2H8) (52) and PE-conjugated hamster anti-mouse TNFR2 antibody (clone TR75-89; BD Biosciences - Pharmingen) at final concentrations of $5 \mu \mathrm{g} / \mathrm{ml}$ and 10 $\mu \mathrm{g} / \mathrm{ml}$, respectively. Tissues were imaged with a Nikon Eclipse TE2000$\mathrm{U}$ inverted microscope, using a 20x PlanApo lens and a Bio-Rad Radiance2100 (Zeiss) laser scanning confocal unit.

Flow cytometry. Infiltrating renal $\mathrm{T}$ cells and macrophages were quantitated by 4-color flow cytometry. Single-cell suspensions from individual kidneys were prepared as described elsewhere (53). For analysis of leukocyte surface markers, $0.5 \times 10^{6}$ to $1 \times 10^{6}$ cells were simultaneously incubated with $1 \mu \mathrm{g}$ of each of the following fluorochrome-conjugated monoclonal antibodies: allophycocyanin-conjugated hamster anti-mouse CD3 $\varepsilon$ (clone 145-2C11; BD Biosciences - Pharmingen), PE-Cy5-conjugated hamster anti-mouse TCR $\beta$ chain (clone H57-597; BD Biosciences - Pharmingen), PE-conjugated hamster anti-mouse $\gamma \delta$ TCR (clone GL3; BD Biosciences Pharmingen), and FITC-conjugated rat anti-mouse F4/80 (clone CL:A3-1; Serotec). The amounts of positively stained $\alpha \beta$ T cells, $\gamma \delta$ T cells, and macrophages were expressed as percentage of total renal cells.

TNFR1 expression on leukocytes was characterized by flow cytometry using a monoclonal hamster anti-mouse TNFR1 antibody (clone 55R286; BD Biosciences - Pharmingen), followed by a biotin-labeled mouse anti-hamster IgG cocktail (BD Biosciences - Pharmingen). Cells were then stained with PE-conjugated streptavidin (DakoCytomation) and either allophycocyanin-conjugated anti-CD3e, PE-Cy5-conjugated anti-TCR $\beta$ chain and FITC-conjugated anti- $\gamma \delta$ TCR (BD Biosciences - Pharmingen) or allophycocyanin-conjugated anti-mouse macrophage clone F4/80 (Serotec). Hamster IgG1 (BD Biosciences - Pharmingen) was used as the appropriate isotype control. TNFR2 expression on leukocytes was analyzed with a PE-conjugated monoclonal hamster anti-mouse TNFR2 antibody (clone TR75-89; BD Biosciences - Pharmingen). PE-conjugated hamster IgG1 was used as isotype control.

Flow cytometric detection of apoptotic $\alpha \beta$ T cells was performed by staining single-cell suspensions obtained from spleen, lymph nodes, and kidneys with allophycocyanin-conjugated anti-mouse CD3e, PE-Cy5-conjugated anti-mouse TCR $\beta$ chain, and annexin V-FITC (BD Biosciences - Pharmingen). Propidium iodide (BD Biosciences - Pharmingen) staining was used to exclude dead cells.

Statistical analysis. Results are expressed as mean \pm SEM or SD as indicated. Significance of differences was determined by ANOVA, followed by Bonferroni's post-hoc test for multiple comparisons when appropriate. $P<0.05$ was considered statistically significant.

\section{Acknowledgments}

We thank Ling Xiao for technical assistance in BM preparations and measurement of proteinuria and Peter O'Donnell for confocal imaging. We are grateful to Junichi Hirahashi for valuable discussions. We thank William Muller (Department of Pathology, Weill Medical College of Cornell University, New York, New York, USA) for generously providing Alexa 488-conjugated antimouse CD31/PECAM-1 antibody. This research was supported by NIH grants RO1 DK-51643 and PO1 HL-36028 to T.N. Mayadas and Deutsche Forschungsgemeinschaft research grant Vi 231/1-1 to V. Vielhauer.

Received for publication September 14, 2004, and accepted in revised form February 1, 2005.

Address Correspondence to: Tanya N. Mayadas, Department of Pathology, Center of Excellence in Vascular Biology, Brigham and Women's Hospital and Harvard Medical School, NRB 0752O, 77 Avenue Louis Pasteur, Boston, Massachusetts 02115, USA. Phone: (617) 525-4336; Fax: (617) 525-4333; E-mail: tmayadas@rics.bwh.harvard.edu. 
1. Couser, W.G., and Neilson, E.G. 2001. Overview of renal inflammation. In Immunologic renal diseases. E.G. Neilson and W.G. Couser, editors. Lippincott Williams \& Wilkins. Philadelphia, Pennsylvania, USA. 47-53.

2. Pusey, C.D., and Rees, A.J. 1998. Rapidly progressive glomerulonephritis. Oxford University Press. Oxford, United Kingdom. 296 pp.

3. Lan, H.Y. 1998. Therapeutic effects of cytokine blockade in glomerulonephritis. Nephrol. Dial. Transplant. 13:7-9.

4. Bertani, T., et al. 1989. Tumor necrosis factor induces glomerular damage in the rabbit. Am.J. Pathol. 134:419-430.

5. Tomosugi, N.I., et al. 1989. Modulation of antibody-mediated glomerular injury in vivo by bacterial lipopolysaccharide, tumor necrosis factor, and IL-1. J. Immunol. 142:3083-3090.

6. Le Hir, M., Haas, C., Marino, M., and Ryffel, B. 1998. Prevention of crescentic glomerulonephritis induced by anti-glomerular membrane antibody in tumor necrosis factor-deficient mice. Lab. Invest. 78:1625-1631.

7. Ryffel, B., Eugster, H., Haas, C., and Le Hir, M. 1998. Failure to induce anti-glomerular basement membrane glomerulonephritis in TNF alpha/beta deficient mice. Int. J. Exp. Pathol. 79:453-460.

8. Karkar, A.M., et al. 1992. Passive immunization against tumour necrosis factor-alpha (TNFalpha) and IL-1 beta protects from LPS enhancing glomerular injury in nephrotoxic nephritis in rats. Clin. Exp. Immunol. 90:312-318.

9. Lan, H.Y., et al. 1997. TNF-alpha up-regulates renal MIF expression in rat crescentic glomerulonephritis. Mol. Med. 3:136-144.

10. Karkar, A.M., Smith, J., and Pusey, C.D. 2001. Prevention and treatment of experimental crescentic glomerulonephritis by blocking tumour necrosis factor-alpha. Nephrol. Dial. Transplant. 16:518-524.

11. Aggarwal, B.B., Samanta, A., and Feldmann, M. 2001. TNFa. In Cytokine reference. J.J. Oppenheim and M. Feldmann, editors. Academic Press. San Diego, California, USA. 415-463.

12. Baud, L., et al. 1989. Production of tumor necrosis factor by rat mesangial cells in response to bacterial lipopolysaccharide. Kidney Int. 35:1111-1118.

13. Gomez-Guerrero, C., Lopez-Armada, M.J., Gonzalez, E., and Egido, J. 1994. Soluble IgA and IgG aggregates are catabolized by cultured rat mesangial cells and induce production of TNFalpha and IL-6, and proliferation. J. Immunol. 153:5247-5255.

14. Neale, T.J., et al. 1995. Tumor necrosis factor-alpha is expressed by glomerular visceral epithelial cells in human membranous nephropathy. Am.J. Pathol. 146:1444-1454.

15. Wuthrich, R.P., et al. 1990. MHC class II, antigen presentation and tumor necrosis factor in renal tubular epithelial cells. Kidney Int. 37:783-792.

16. Jevnikar, A.M., et al. 1991. Stimulated kidney tubular epithelial cells express membrane associated and secreted TNF alpha. Kidney Int. 40:203-211.

17. Timoshanko, J.R., Sedgwick, J.D., Holdsworth, S.R., and Tipping, P.G. 2003. Intrinsic renal cells are the major source of tumor necrosis factor contributing to renal injury in murine crescentic glomerulonephritis. J. Am. Soc. Nephrol. 14:1785-1793.

18. Vassalli, P. 1992. The pathophysiology of tumor necrosis factors. Annu. Rev. Immunol. 10:411-452.
19. Feldmann, M., Brennan, F.M., and Maini, R. 1998. Cytokines in autoimmune disorders. Int. Rev. Immunol. 17:217-228.

20. Kassiotis, G., and Kollias, G. 2001. Uncoupling the proinflammatory from the immunosuppressive properties of tumor necrosis factor (TNF) at the p55 TNF receptor level: implications for pathogenesis and therapy of autoimmune demyelination. J. Exp. Med. 193:427-434.

21. Kollias, G., and Kontoyiannis, D. 2002. Role of TNF/ TNFR in autoimmunity: specific TNF receptor blockade may be advantageous to anti-TNF treatments. Cytokine Growth Factor Rev. 13:315-321.

22. Speiser, D.E., et al. 1996. Tumor necrosis factor receptor $\mathrm{p} 55$ mediates deletion of peripheral cytotoxic $\mathrm{T}$ lymphocytes in vivo. Eur. J. Immunol. 26:3055-3060.

23. Al Lamki, R.S., et al. 2001. Expression of tumor necrosis factor receptors in normal kidney and rejecting renal transplants. Lab. Invest. 81:1503-1515.

24. Aten, J., et al. 2000. Strong and selective glomerular localization of CD134 ligand and TNF receptor-1 in proliferative lupus nephritis. J. Am. Soc. Nephrol. 11:1426-1438

25. Barbara, J.A., et al. 1994. Dissociation of TNFalpha cytotoxic and proinflammatory activities by $\mathrm{p} 55$ receptor- and $\mathrm{p} 75$ receptor-selective TNFalpha mutants. EMBOJ. 13:843-850.

26. Pfeffer, K. 2003. Biological functions of tumor necrosis factor cytokines and their receptors. Cytokine Growth Factor Rev. 14:185-191.

27. Lucas, R., et al. 1998. Both TNF receptors are required for direct TNF-mediated cytotoxicity in microvascular endothelial cells. Eur. J. Immunol. 28:3577-3586

28. Declercq, W., Denecker, G., Fiers, W., and Vandenabeele, P. 1998. Cooperation of both TNF receptors in inducing apoptosis: involvement of the TNF receptor-associated factor binding domain of the TNF receptor 75. J. Immunol. 161:390-399.

29. Pinckard, J.K., Sheehan, K.C., and Schreiber, R.D. 1997. Ligand-induced formation of p55 and p75 tumor necrosis factor receptor heterocomplexes on intact cells. J. Biol. Chem. 272:10784-10789.

30. Tartaglia, L.A., Pennica, D., and Goeddel, D.V. 1993. Ligand passing: the $75-\mathrm{kDa}$ tumor necrosis factor (TNF) receptor recruits TNF for signaling by the 55 kDa TNF receptor. J. Biol. Chem. 268:18542-18548.

31. Grell, M., Becke, F.M., Wajant, H., Mannel, D.N., and Scheurich, P. 1998. TNF receptor type 2 mediates thymocyte proliferation independently of TNF receptor type 1. Eur. J. Immunol. 28:257-263.

32. Lucas, R., et al. 1997. Crucial role of tumor necrosis factor (TNF) receptor 2 and membrane-bound TNF in experimental cerebral malaria. Eur. J. Immunol. 27:1719-1725.

33. Rosenkranz, A.R., Mendrick, D.L., Cotran, R.S., and Mayadas, T.N. 1999. P-selectin deficiency exacerbates experimental glomerulonephritis: a protective role for endothelial P-selectin in inflammation. J. Clin. Invest. 103:649-659.

34. Rosenkranz, A.R., et al. 2000. Regulatory interactions of $\alpha \beta$ and $\gamma \delta$ T cells in glomerulonephritis. Kidney Int. 58:1055-1066.

35. Boyce, N.W., and Holdsworth, S.R. 1986. Evidence for direct renal injury as a consequence of glomerular complement activation. J. Immunol. 136:2421-2425.

36. Quigg, R.J. 2003. Complement and the kidney. J. Immunol. 171:3319-3324.
37. Madge, L.A., Sierra-Honigmann, M.R., and Pober, J.S. 1999. Apoptosis-inducing agents cause rapid shedding of tumor necrosis factor receptor 1 (TNFR1). A nonpharmacological explanation for inhibition of TNF-mediated activation. J. Biol. Chem. 274:13643-13649.

38. Jacobs, M., Brown, N., Allie, N., Chetty, K., and Ryffel, B. 2000. Tumor necrosis factor receptor 2 plays a minor role for mycobacterial immunity. Pathobiology. 68:68-75.

39. Hindmarsh, E.J., and Marks, R.M. 1998. Complement activation occurs on subendothelial extracellular matrix in vitro and is initiated by retraction or removal of overlying endothelial cells. J. Immunol. 160:6128-6136.

40. Le Hir, M., et al. 1996. Differentiation of follicular dendritic cells and full antibody responses require tumor necrosis factor receptor-1 signaling. J. Exp. Med. 183:2367-2372.

41. Zhou, T., et al. 1996. Greatly accelerated lymphadenopathy and autoimmune disease in lpr mice lacking tumor necrosis factor receptor I. J. Immunol. 156:2661-2665.

42. Zhang, H.G., et al. 2000. Antigen presenting cells expressing Fas ligand down-modulate chronic inflammatory disease in Fas ligand-deficient mice. J. Clin. Invest. 105:813-821.

43. Pender, M.P. 1999. Activation-induced apoptosis of autoreactive and alloreactive $\mathrm{T}$ lymphocytes in the target organ as a major mechanism of tolerance. Immunol. Cell Biol. 77:216-223.

44. Radeke, H.H., et al. 1994. Activation of autoreactive T-lymphocytes by cultured syngeneic glomerular mesangial cells. Kidney Int. 45:763-774.

45. Ortiz-Arduan, A., et al. 1996. Regulation of Fas and Fas ligand expression in cultured murine renal cells and in the kidney during endotoxemia. Am.J. Physiol. 271:F1193-F1201.

46. Lorz, C., et al. 2000. Proapoptotic Fas ligand is expressed by normal kidney tubular epithelium and injured glomeruli. J. Am. Soc. Nephrol. 11:1266-1277.

47. Pfeffer, K., et al. 1993. Mice deficient for the 55 $\mathrm{kd}$ tumor necrosis factor receptor are resistant to endotoxic shock, yet succumb to L. monocytogenes infection. Cell. 73:457-467.

48. Erickson, S.L., et al. 1994. Decreased sensitivity to tumour-necrosis factor but normal T-cell development in TNF receptor-2-deficient mice. Nature. 372:560-563.

49. Mombaerts, P., et al. 1992. Mutations in T-cell antigen receptor genes alpha and beta block thymocyte development at different stages. Nature. 360:225-231.

50. Schrijver, G., et al. 1990. Anti-GBM nephritis in the mouse: role of granulocytes in the heterologous phase. Kidney Int. 38:86-95.

51. de Jong, J.P., Leenen, P.J., Voerman, J.S., van der Sluijs-Gelling, A.J., and Ploemacher, R.E. 1994. A monoclonal antibody (ER-HR3) against murine macrophages. II. Biochemical and functional aspects of the ER-HR3 antigen. Cell Tissue Res. 275:577-585

52. Xie, Y., and Muller, W.A. 1993. Molecular cloning and adhesive properties of murine platelet/ endothelial cell adhesion molecule 1. Proc. Natl. Acad. Sci. U. S. A. 90:5569-5573.

53. Vielhauer, V., et al. 2003. Phenotyping renal leukocyte subsets by four-color flow cytometry: characterization of chemokine receptor expression. Nephron Exp. Nephrol. 93:e63-e71. 